\title{
Structure and Physical Properties of Naphthalene Containing Polyesters I. Structure of Poly(butylene 2,6-naphthalate) and Poly(ethylene 2,6-naphthalate) as Studied by Solid State NMR Spectroscopy
}

\author{
Takeshi Yamanobe, Hideo Matsuda, Katsue Imai, Akihiko Hirata, \\ Shinichiro Mori, ${ }^{*}$ and Tadashi Komoto \\ Department of Chemistry, Gunma University, Tenjin-cho, Kiryu, Gunma 376, Japan \\ * NKK Corporation, Materials Processing Research Center, Minamiwatarida, \\ Kawasaki-ku, Kawasaki, Kanagawa 210, Japan
}

(Received November 17, 1995)

\begin{abstract}
C}$ NMR measurements were carried out for poly(ethylene 2,6-naphthalate) (PEN) and poly(butylene 2,6-naphthalate) (PBN) in the solid state in order to investigate the effect of drawing on their structures of PEN and PBN. NMR spectra of undrawn, pellet and melt quenched PBN's were very similar to one another. From the results of DSC and the half time of crystallization measurements, it was found that the rate of crystallization of PBN is so fast that the crystalline phase is formed in the PBN samples. Drawing of PEN and PBN films caused down field shifts of chemical shifts for OCH${ }_{2}$, $\mathrm{CH}_{2}$ and $\mathrm{C} 4 \mathrm{a}, 8 \mathrm{a}$ carbons. For PEN, the structural change from amorphous to $\alpha$ phase takes place by drawing. Chemical shift change arose from the structural change from amorphous to $\alpha$ phase. For PBN, the crystal structural change from $\alpha$ to $\beta$ phases occurs. It was concluded that the change in crystal structure caused by drawing leads to the down field shift of chemical shift for PBN.

KEY WORDS Poly(ethylene 2,6-naphthalate) / Poly(butylene 2,6-naphthalate) / Cross Polarization Magic Angle Spinning Nuclear Magnetic Resonance (CPMAS NMR) / Differential Scanning Calorimetry (DSC) / Drawing / Crystallization /
\end{abstract}

Poly(ethylene terephthalate) (PET) is one of widely used polymers because of its superior physical and chemical properties at ambient temperatures due to glass transition temperature $T_{\mathrm{g}}$ of $\mathrm{ca} .69^{\circ} \mathrm{C}$, melting temperature $c a .267^{\circ} \mathrm{C}$ and chemical resistance to most organic solvents and aqueous solutions. Interests have recently become paid to other polyesters such as poly(butylene terephthalate) (PBT) $\left(T_{\mathrm{g}}=60^{\circ} \mathrm{C}, T_{\mathrm{m}}=\right.$ $228^{\circ} \mathrm{C}$ ), poly(ethylene 2,6-naphthalate) (PEN) $\left(T_{\mathrm{g}}=\right.$ $114^{\circ} \mathrm{C}, T_{\mathrm{m}}=260^{\circ} \mathrm{C}$ ), and poly(butylene 2,6-naphthalate) $(\mathrm{PBN})\left(T_{\mathrm{g}}=41^{\circ} \mathrm{C}, T_{\mathrm{m}}=240^{\circ} \mathrm{C}\right)$.

Compared with PET, PBT having more flexible butylene unit undergoes a unique reversible crystalline $\alpha$ to $\beta$ phase transition by uniaxial drawing at room temperature. ${ }^{1,2}$ From ${ }^{13} \mathrm{C}$ CPMAS NMR study, it is reported that the driving force for the transition results from the packing efficiency of the aromatic rings in the $\alpha$ phase. $^{3}$ The population of trans conformation of terephthaloyl residue is higher for $\beta$ phase than for $\alpha$ phase. $^{4}$

It is of interest that PBN film undergoes a similar crystal phase transition between $\alpha$ and $\beta$ phases when being uniaxially drawn. ${ }^{5}$

Compared with the properties of PET, the high $T_{\mathrm{g}}$ of ca. $114^{\circ} \mathrm{C}$ and the high Young's modulus and superior mechanical strength of PEN is clearly due to the strong interaction of naphthalene rings. On the other hand, PBN has a flexible butylene group and a naphthalene ring comprising a strong interchain interaction in the repeating unit.

It is therefore of interest to investigate the effect of the butylene and naphthalene groups on the structure and properties of polyesters with reference to molecular mobility as studied by solid state NMR. However, a few publications have been reported on structure and properties of PBN. ${ }^{6}$

In this paper, we will report on the structure of drawn and undrawn PEN and PBN by means of solid state NMR and other measurements.

\section{EXPERIMENTAL}

Specimens

(1) Drawn Films. Undrawn PBN films with thickness of $c a .100 \mu \mathrm{m}$, which had been melt extruded to room temperature, were drawn at a rate of $1 \mathrm{mmin}^{-1}$ at $170^{\circ} \mathrm{C}$ to $300 \%(1 \times 3)$ and $360 \%(1 \times 3.6)$ with the fixed width. Undrawn PEN films were also drawn at the same rate at $140^{\circ} \mathrm{C}$ to $300 \%(1 \times 3)$ and $400 \%(1 \times 4)$ with the fixed width.

(2) Melt Quenched PBN. PBN in a glass tube sealed in vacuo was melted at $280^{\circ} \mathrm{C}$ and then quenched in a dry-ice/methanol bath (at about $-70^{\circ} \mathrm{C}$ ).

\section{NMR Measurements}

The ${ }^{13} \mathrm{C}$ CPMAS NMR spectrum was recorded at using a JEOL EX270W spectrometer $(67.5 \mathrm{MHz})$ with a CPMAS accessory. The sample was contained in a cylindrical ceramic rotor. The rotor was spun at about $6 \mathrm{kHz}$. The contact time was $2 \mathrm{~ms}$ and the repetition time was $5 \mathrm{~s}$. The spectral widths were $27 \mathrm{kHz} .{ }^{13} \mathrm{C}$ NMR chemical shifts were calibrated indirectly using the adamantane upfield peak $(29.5 \mathrm{ppm})$ relative to tetramethylsilane.

The dipolar dephasing (DD) spectrum was recorded using a JEOL EX270W spectrometer $(67.5 \mathrm{MHz})$. This method reduces signals of protonated carbons to zero. By this method, only signals of quarternaly carbons can be observed. 


\section{DSC Measurements}

DSC measurements were carried out by using a Perkin Elmer DSC 7. All measurements were done in nitrogen atmosphere. Temperature was calibrated by using In $\left(T_{\mathrm{m}}=156.9^{\circ} \mathrm{C}\right)$ and $\mathrm{Zn}\left(T_{\mathrm{m}}=419.5^{\circ} \mathrm{C}\right)$.

\section{RESULTS AND DISCUSSION}

In Figure 1a is shown a ${ }^{13} \mathrm{C}$ CPMAS spectrum of undrawn PBN film. The peaks at about 26 and $67 \mathrm{ppm}$ are assigned to internal $\mathrm{CH}_{2}$ and $\mathrm{CH}_{2}$ directly bonded to oxygen, respectively. From the chemical shift values, the peak at the lowest field is assigned to the signal of carbonyl carbon of terephthaloyl group. Peaks for the aromatic carbons which appeared in range from 120 to $140 \mathrm{ppm}$ are complex. In Figure $1 \mathrm{~b}$ is shown $\mathrm{a}^{13} \mathrm{C} \mathrm{DD}$ spectrum of the undrawn PBN film, where only three peaks are observed. A peak at the lowest field is assigned to the carbonyl peak as seen in the CPMAS spectrum. According to the assignment for the naphthalene unit reported for $\mathrm{PEN}^{7}$ the upfield peak is assigned to $\mathrm{C} 2$ and C6 carbons which are directly bonded to carbonyl carbons, the numbers of the naphthalene carbons being indicated in the figure. ${ }^{13} \mathrm{C}$ chemical shift values for the undrawn, melt quenched and pellet PBN's are summarized in Table I together with those for PBT. ${ }^{4}$
As the chemical structure of aromatic groups of PBN and PBT is different, it is difficult to compare ${ }^{13} \mathrm{C}$ chemical shift of the aromatic groups. ${ }^{13} \mathrm{C}$ chemical shifts of carbonyl groups and $\mathrm{OCH}_{2}$ of $\mathrm{PBN}$ are in agreement with those of $\mathrm{PBT}$, respectively within the experimental error. This indicates that the conformation around these carbons of PBN is similar to those of PBT.

It is also noted from Table I that ${ }^{13} \mathrm{C}$ chemical shift (25.6 ppm) of interior $\mathrm{CH}_{2}$ of PBN's is close to that (26.6 ppm) of PBT in amorphous state rather than those of PBT in $\alpha$ and $\beta$ phases. From ${ }^{13} \mathrm{C}$ chemical shifts of model compounds for PBT, it has already been reported that interior $\mathrm{CH}_{2}$ of PBT in $\alpha$ and $\beta$ phases takes all trans conformation. ${ }^{4}$ As ${ }^{13} \mathrm{C}$ chemical shift is governed by relatively local electronic structure, ${ }^{8}$ it is worthy to compare ${ }^{13} \mathrm{C}$ chemical shifts of interior $\mathrm{CH}_{2}$ between PBN and PBT. Taking into account $\gamma$-gauche effect, ${ }^{9}$ the higher field shift for the interior $\mathrm{CH}_{2}$ of $\mathrm{PBN}$ than those of PBT in $\alpha$ and $\beta$ phases means that interior $\mathrm{CH}_{2}$ of PBN takes a rapid transition between trans and gauche conformations. Since ${ }^{13} \mathrm{C}$ chemical shifts of interior $\mathrm{CH}_{2}$ for PBN's appear at a field about $1 \mathrm{ppm}$ higher than for amorphous PBT, the interior $\mathrm{CH}_{2}$ for PBN takes gauche rich conformation compared with that for amorphous PBT.

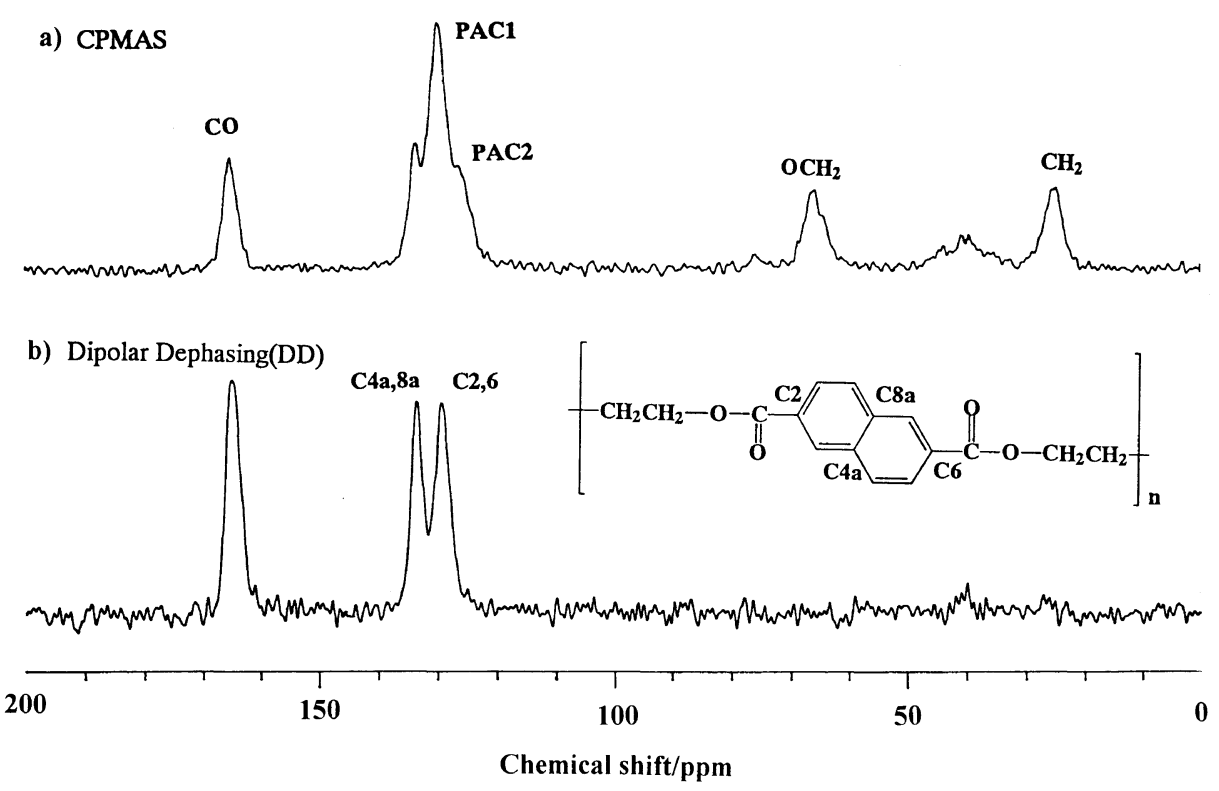

Figure 1. ${ }^{13} \mathrm{C}$ NMR spectra of the undrawn PBN film. a) CPMAS and b) Dipolar Dephasing (DD).

Table I. ${ }^{13} \mathrm{C}$ chemical shifts for undrawn PBN and PBT specimens

\begin{tabular}{|c|c|c|c|c|c|c|c|c|}
\hline Sample & Method & $\mathrm{CO}$ & $\mathrm{C} 4 \mathrm{a}, 8 \mathrm{a}$ & $C 2,6^{\mathrm{a}}$ & $\mathrm{PACl}$ & PAC2 & $\mathrm{OCH}_{2}$ & $\mathrm{CH}_{2}$ \\
\hline \multicolumn{9}{|l|}{ PBN } \\
\hline \multirow[t]{2}{*}{ Pellet } & CPMAS & 165.9 & 133.7 & & 130.2 & 126.6 & 66.8 & 25.6 \\
\hline & DD & 166.0 & 133.9 & 129.6 & & & & \\
\hline \multirow[t]{2}{*}{ Melt quench } & CPMAS & 166.0 & 133.8 & & 130.1 & 126.7 & 66.9 & 25.6 \\
\hline & DD & 166.0 & 133.9 & 129.9 & & & & \\
\hline \multirow[t]{2}{*}{ Undrawn } & CPMAS & 165.7 & 133.6 & & 130.0 & 126.8 & 66.6 & 25.8 \\
\hline & DD & 165.9 & 134.0 & 129.7 & & & & \\
\hline \multicolumn{9}{|l|}{$\mathrm{PBT}^{4}$} \\
\hline$\alpha$ & CPMAS & 165.5 & & 135.2 & 130.8 & & 66.2 & 27.2 \\
\hline$\beta$ & CPMAS & 165.5 & & 135.2 & 131.7 & & 66.7 & 27.6 \\
\hline Amorphous & CPMAS & 166.0 & & 134.9 & 130.2 & & 65.9 & 26.6 \\
\hline
\end{tabular}

${ }^{a} \mathrm{C} 2,6$ denotes carbon atoms of naphthalene group of PBN. Carbons for PBT are $\mathrm{C} 1$ and $\mathrm{C} 4$ of its benzene group. 
It is of great interest that ${ }^{13} \mathrm{C}$ chemical shift of any carbon atom is almost same among pellet, melt quenched, and undrawn PBN's. This may mean that structure of PBN is independent of the process by which PBN specimen was prepared, although the structure of most crystalline polymers is governed by crystallization conditions. In order to clarify the reason for no structure difference among the specimens, rate of crystallization from melt was measured as function of crystallization temperature, $T_{\mathrm{c}}$. Figure 2 shows half time $\tau_{1 / 2}$ of crystallization against $T_{\mathrm{c}}$ for PET, PEN, and PBN. In cases of PET and PEN, $\tau_{1 / 2}$ took a minimum at $180^{\circ} \mathrm{C}$ and $200^{\circ} \mathrm{C}$, respectively, indicating the maximum rate of crystallization occurred at these temperatures. On the other hand, $\tau_{1 / 2}$ for PBN decreases monotonously with decreasing $T_{\mathrm{c}}$, indicating that the rate of crystallization for PBN increased with decreasing $T_{\mathrm{c}}$. This may mean that quenching from the melt can not produce amorphous PBN but crystalline one. In other words, the very fast rate of crystallization of $\mathrm{PBN}$ is the reason why ${ }^{13} \mathrm{C}$ chemical shift of the corresponding carbon atom is almost same among the specimens.

In order to confirm the very fast crystallization, DSC measurements were also carried out for the undrawn PBN and PEN specimens. In Figure 3 are shown DSC thermograms of the undrawn PEN and PBN films in heating and cooling processes. The heating and cooling rates were $20^{\circ} \mathrm{C} \mathrm{min}^{-1}$. For the undrawn PEN, a change in baseline slope was observed in the heating process at about $118^{\circ} \mathrm{C}$ which corresponds to $T_{\mathrm{g}}$ (Figure $3 \mathrm{a}$ )). Exothermic and endothermic peaks appeared at 200 230 and $256^{\circ} \mathrm{C}$, respectively. This thermogram clearly shows a cold crystallization and melting process. It is also noted that heat of the cold crystallization $\Delta H_{1}$ of this exotherm is almost equal to heat of melt-

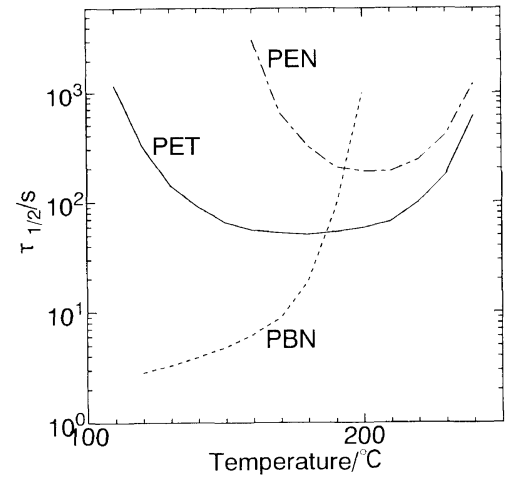

Figure 2. Crystallization temperature dependences of half time $\tau_{1 / 2}$ of crystallization for PET, PEN, and PBN.

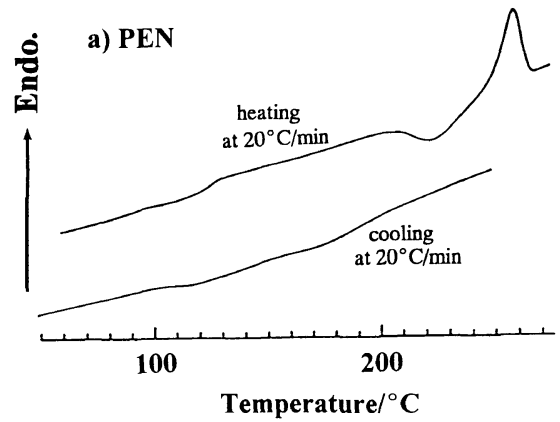

ing $\Delta H_{2}$ of the successive endotherm. It is said therefore that the amorphous PEN chains having $T_{\mathrm{g}}$ of $118^{\circ} \mathrm{C}$ underwent a cold crystallization just above $200^{\circ} \mathrm{C}$ and then melted at $250^{\circ} \mathrm{C}$. However, no exothermic peak was observed in the cooling process from the melt (Figure 3a)). This means that PEN chain cooled down to room temperature at $20^{\circ} \mathrm{C} \mathrm{min}^{-1}$ were frozen as if being melt-quenched.

On the other hand, only an endothermic peak was observed in DSC thermogram of the undrawn PBN (Figure $3 b)$ ). $T_{\mathrm{g}}$ was observed at about $41^{\circ} \mathrm{C}$ as a slight change of baseline slope (not shown), which is close to the reported data, ${ }^{2} 48^{\circ} \mathrm{C}$. There is no exotherm corresponding cold crystallization, suggesting that most parts of the undrawn $\mathrm{PBN}$ chains are crystalline. A sharp exotherm was observed at $200^{\circ} \mathrm{C}$ in cooling process from the melt (Figure $3 b$ )). This means PBN chains are not quenched into an amorphous state but into the crystalline state even at a cooling rate of $20^{\circ} \mathrm{C} \mathrm{min.}^{-1}$

It was revealed from these results that the crystallization rate of $\mathrm{PBN}$ is quite fast compared with the other polyesters. A strong interchain interaction between naphthalene groups may cause interchain association during cooling from the melt. Rotations of both the naphthalene and ethylene units of PEN seem to be restricted so that PEN chains are easily frozen into amorphous state by quenching from melt. On the other hand, it can be said that the long butylene units contained in PBN chains interrupt an association of the naphthalene groups to some extent as compared with PEN having short ethylene units and that the butylene unit is more flexible than the ethylene unit. Thus, the high chain mobility of PBN may give a fast nucleation and growth of PBN crystals from melt. This chemical structure difference should give rise to a fast crystallization of PBN from melt, while PEN chain are not so mobile that they may be frozen into amorphous state even at a cooling rate of $20^{\circ} \mathrm{C} \mathrm{min.}^{-1}$

In Figure 4 are shown ${ }^{13} \mathrm{C}$ CPMAS spectra of undrawn and drawn $(1 \times 3,1 \times 3.6) \mathrm{PBN}$ films. Comparing the spectra of drawn samples with that of undrawn one, a difference is found at peaks assigned to the naphthalene group. The upper field shoulder peak of naphthalene group in undrawn PBN film is very weak, while it is a little bit strong in both drawn PBN films so that the peak tops are easy to be found out. This fact seems to be relevant to a chemical shift difference between the undrawn and drawn films. ${ }^{13} \mathrm{C}$ chemical shifts of the undrawn and drawn PBN and PEN films are summarized in Table II.

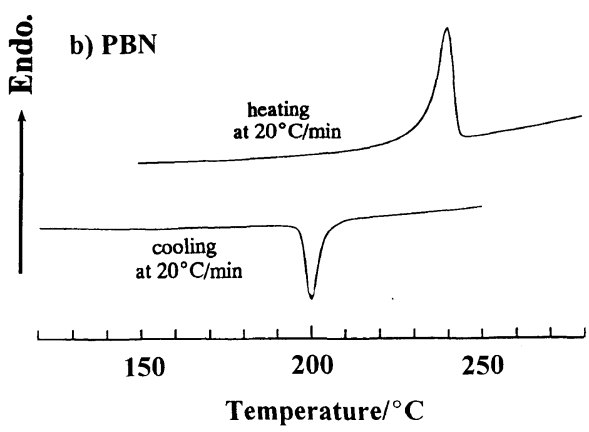

Figure 3. DSC thermograms of PEN and PBN. a) PEN and b) PBN. 
${ }^{13} \mathrm{C}$ chemical shifts of carbonyl carbon are almost same for all $\mathrm{PBN}$ and PEN films within the experimental error as is evident from Table II. This means that the conformation around the ester $\mathrm{CO}$ group is independent of drawing condition.

${ }^{13} \mathrm{C}$ chemical shifts of $\mathrm{CH}_{2}$ for both drawn PBN films appeared at a lower field than undrawn one. The low field shift due to $\gamma$-gauche effect indicates a high population of trans conformation for $\mathrm{CH}_{2}$ carbons. As $\gamma$ position to the $\mathrm{CH}_{2}$ carbon is carbonyl carbon and a oxygen atom bonded to carbonyl carbon, the trans conformation of $\mathrm{OCH}_{2}-\mathrm{CH}_{2}$ and $\mathrm{O}-\mathrm{CH}_{2}$ bonds increased in amount by drawing. It is also found that the internal $\mathrm{CH}_{2}$ chemical shift for $1 \times 3.6 \mathrm{PBN}$ film appeared at a field $(26.6 \mathrm{ppm}) \mathrm{ca} .0 .3 \mathrm{ppm}$ lower than for $1 \times 3$ PBN film $(26.3 \mathrm{ppm})$. This indicates that the trans population increases with increasing draw ratio.

A similar trend was observed for $\mathrm{OCH}_{2} \cdot{ }^{13} \mathrm{C}$ chemical shifts of $\mathrm{OCH}_{2}$ for drawn $(1 \times 3$ and $1 \times 3.6) \mathrm{PBN}$ films appeared at lower field $(67.3$ and $67.2 \mathrm{ppm}$, respectively) than that of undrawn PBN film $(66.6 \mathrm{ppm})$. On the contrary, ${ }^{13} \mathrm{C}$ chemical shifts of drawn PEN films moves to upper field. As the number of methylene unit is 2 and 4 for PEN and PBN, respectively, direct comparison of chemical shifts for this group seems to be difficult. It is worthy of note that ${ }^{13} \mathrm{C}$ chemical shift of $\mathrm{OCH}_{2}$ of PBN changed to lower fields by drawing, while it changed to higher fields in case of PEN. It can be said
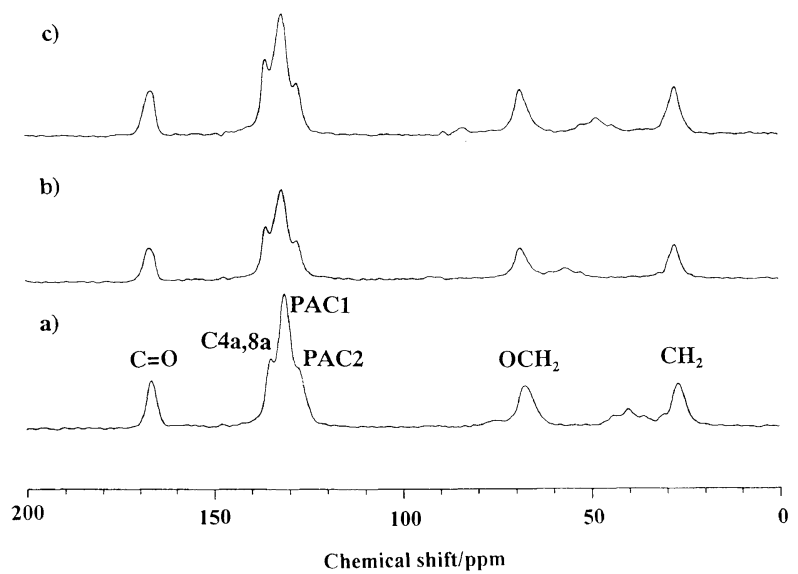

Figure 4. ${ }^{13} \mathrm{C}$ CPMAS NMR spectra of PBN films. a) undrawn, b) drawn $(1 \times 3)$, and c) drawn $(1 \times 3.6)$. that a structural change did occur around this group.

A small effect of drawing on ${ }^{13} \mathrm{C}$ chemical shift for protonated aromatic carbons (PAC1 and PAC2), C2 and C6 carbons was found (Table II). A detailed chemical shift change by drawing may be covered by broadening of these peaks. It should be noted that ${ }^{13} \mathrm{C}$ chemical shift differences for $\mathrm{C} 4 \mathrm{a}$ and $\mathrm{C} 8 \mathrm{a}$ between undrawn and drawn PBN films are as large as $0.5-1.0 \mathrm{ppm}$ which are larger than any other carbons. Similar chemical shift changes to lower field by $1.3-1.5 \mathrm{ppm}$ by drawing were found for $\mathrm{C} 4 \mathrm{a}$ and $\mathrm{C} 8 \mathrm{a}$ of PEN films. This change is larger than those of PBN's.

It is of importance to investigate a relationship of the above obtained chemical shift changes with the morphology of the undrawn and drawn films. From transmission electron microscopy of an ultrathin cross-section of the drawn PEN films, we found that crystalline phases were grown perpendicular to the drawing direction (machine direction). ${ }^{10}$ This morphology was not observed for undrawn PEN film. As was found from the DSC thermogram, undrawn PEN film is nearly amorphous. This result is consistent with an X-ray study ${ }^{11}$ that a drawn PEN film takes the crystalline $\alpha$ phase structure. It was also revealed from our dichroic micro-FT IR measurement of ultrathin crosssection that the plane of naphthalene rings is aligned parallel to the drawn PEN film surface..$^{10}$ It is, therefore, concluded that the structural change of PEN from the amorphous state to the oriented $\alpha$ phases is relevant to the chemical shift changes for $\mathrm{C} 4 \mathrm{a}$ and $\mathrm{C} 8 \mathrm{a}$ of the naphthalene ring.

There may be two possibilities for these chemical shift changes for PEN film due to drawing: 1) conformational change and 2) crystal structure change. As was mentioned above, the conformational change for $\mathrm{CH}_{2}$ and $\mathrm{OCH}_{2}$ from gauche to trans conformation due to molecular chain orientation by drawing is predominant. As C4a and $\mathrm{C} 8 \mathrm{a}$ are quaternary carbons positioned at center of naphthalene ring, there should be no conformational effect on their chemical shift change by drawing. Thus, the chemical shift changes for these C4a and C8a by drawing is only accounted for by the structural change such that the naphthalene rings are aligned parallel in oriented crystalline phase formed by drawing at $140^{\circ} \mathrm{C}$.

It was revealed from DSC thermograms that the

Table II. ${ }^{13} \mathrm{C}$ chemical shifts for drawn and undrawn PBN and PEN specimens

\begin{tabular}{|c|c|c|c|c|c|c|c|c|}
\hline Sample & Method & $\mathrm{CO}$ & $\mathrm{C} 4 \mathrm{a}, 8 \mathrm{a}$ & $\mathrm{C} 2,6$ & $\mathrm{PACl}$ & PAC2 & $\mathrm{OCH}_{2}$ & $\mathrm{CH}_{2}$ \\
\hline \multicolumn{9}{|l|}{ PBN } \\
\hline \multirow{2}{*}{ Undrawn } & CPMAS & 165.7 & 133.6 & & 130.0 & 126.8 & 66.6 & 25.8 \\
\hline & DD & 165.9 & 134.0 & 129.7 & & & & \\
\hline \multirow[t]{2}{*}{$1 \times 3$} & CPMAS & 166.2 & 134.5 & & 130.6 & 127.0 & 67.3 & 26.3 \\
\hline & DD & 166.1 & 134.7 & 130.0 & & & & \\
\hline \multirow[t]{2}{*}{$1 \times 3.6$} & CPMAS & 165.9 & 134.5 & & 130.5 & 126.5 & 67.2 & 26.6 \\
\hline & DD & 166.1 & 134.6 & 130.5 & & & & \\
\hline \multicolumn{9}{|l|}{ PEN } \\
\hline \multirow{2}{*}{ Undrawn } & CPMAS & 165.8 & & & 129.4 & & 63.2 & \\
\hline & DD & 166.0 & 133.8 & 129.5 & & & & \\
\hline \multirow[t]{2}{*}{$1 \times 3$} & CPMAS & 166.1 & & & 129.8 & & 61.7 & \\
\hline & DD & 165.9 & 135.1 & 129.8 & & & & \\
\hline \multirow{2}{*}{$1 \times 4$} & CPMAS & 166.1 & & & 129.9 & & 61.6 & \\
\hline & DD & 166.1 & 135.3 & 129.7 & & & & \\
\hline
\end{tabular}


crystalline phase exists in undrawn PBN film. The corresponding chemical shift change by drawing can not be due to a structural change from amorphous to oriented crystalline phases. It has already been reported that there are two crystalline phases, $\alpha$ and $\beta$, for $\mathrm{PBN}^{5}$ where the transition from the $\alpha$ to $\beta$ phase of PBN takes place by drawing. As the fiber repeat distance of $\alpha$ phase is shorter than that of $\beta$ phase, the conformation of butylene unit is different between them. ${ }^{5}$ The chemical shift change observed for $\mathrm{PBN} \mathrm{CH}_{2}$ and $\mathrm{OCH}_{2}$ carbons may arise from this conformational change. Similarly to the case of PEN, there should be no conformational effect on chemical shift change for C4a and C8a of PBN. From electron microscopy we obtained a morphological change of PBN film by drawing, although an ill-defined oriented crystal phase was grown perpendicular to the machine direction. ${ }^{10}$ A dichroic miro FT IR study also revealed a parallel alignment of naphthalene ring plane to film suface by drawing. ${ }^{10}$ Therefore, it is said that the change of crystal structure from $\alpha$ to $\beta$ phases, especially the change of interaction between naphthalene rings, caused the chemical shift changes. From these results, it can be concluded that the phase transition between $\alpha$ and $\beta$ gave rise to the chemical shift changes of PBN.

Concerning an effect of crystal structure on chemical shift values, two factors can be considered. One is a change in electronic structure and the other an effect of anisotropy of magnetic susceptibility. The first factor has been reported for polyethylene and $n$-paraffins that a difference of crystal structure between orthorhombic and triclinic unit cell results in about $1 \mathrm{ppm}$ chemical shift change. ${ }^{12,13}$ Although the interchain distance of PBN and PEN are longer than that of polyethylene, the monomer unit is bulky for PBN and PEN as compared with polyethylene. The effect of electronic structure may be estimated to be similar to that of polyethylene. (For a quantitative estimation, a quantum chemical calculation such as tight binding method ${ }^{14}$ is required.)

In order to estimate a chemical shift change by the second factor, a source of anisotropic magnetic susceptibility is required. Such a source causes chemical shift change based on analogies to ring current shifts. An orientation dependent magnetic susceptibility can be replaced by magnetic dipoles. The local field at a given site resulting from such a dipole depends on the inverse cube of the distance to the dipole. If the environment surrounding an aromatic group in a polymer chain is approximated by neighboring four chains $4.2 \AA$ (lattice constant of orthorhombic polyethylene) distant, the chemical shift change in going from perpendicular arrangement to parallel arrangement is estimated to be about $1.3 \mathrm{ppm}$ down field shift from the distance dependence of the dipolar field. ${ }^{15}$ Since the interchain distance is longer for PEN and PBN than polyethylene, chemical shift change expected by the rearrangement of neighboring chains would be small for PEN and PBN.
From estimation by both factors, the chemical shift changes of C4a and $\mathrm{C} 8 \mathrm{a}$ in going from undrawn to drawn PEN and PBN films may be affected by both factors. As the crystalline morphology revealed by electron microscopy is ill-defined for PBN than PEN, i.e., the polymer chain arrangement is less ordered in oriented PBN film than PEN, the chemical shift change of PBN may be less than that of PEN.

\section{CONCLUSIONS}

1) For melt quenched, pellet and undrawn PBN films, ${ }^{13} \mathrm{C}$ chemical shifts of all the corresponding carbon atoms are in agreement with one another within the experimental error. From DSC measurements, it was found that the rate of crystallization of $\mathrm{PBN}$ is extremely fast and this results in no chemical shift difference among the specimens.

2) ${ }^{13} \mathrm{C}$ chemical shifts of $\mathrm{CH}_{2}, \mathrm{OCH}_{2}$, and $\mathrm{C} 4 \mathrm{a}, 8 \mathrm{a}$ for PEN were changed by drawing. These chemical shift changes are attributed to the structral change from amorphous to crystalline $\alpha$ phase.

3) ${ }^{13} \mathrm{C}$ chemical shifts of $\mathrm{CH}_{2}, \mathrm{OCH}_{2}$, and $\mathrm{C} 4 \mathrm{a}, 8 \mathrm{a}$ for $\mathrm{PBN}$ moved to down field shift by drawing. Down field shifts of $\mathrm{CH}_{2}$ and $\mathrm{OCH}_{2}$ are caused by increased population of trans conformation in butylene unit. The change of interchain interaction according to phase transiton from $\alpha$ to $\beta$ phases results in the down field shift of $\mathrm{C} 4 \mathrm{a}, 8 \mathrm{a}$.

\section{REFERENCES}

1. C. A. Boye and J. R. Overton, Bull. Am. Phys. Soc., 19, 352 (1974).

2. R. Jakeways, I. M. Ward, M. A. Wilding, I. H. Hall, I. J. Desborough, and M. G. Pass, J. Polym. Sci., Polym. Phys. Ed., 13, 799 (1975).

3. B. C. Perry, J. L. Koening, and J. B. Lando, Macromolecules, 20, 422 (1987).

4. M. A. Gomez, M. H. Cozine, and A. E. Tonelli, Macromolecules, 21, 388 (1988).

5. H. Watanabe, Koubunshi Ronbunshu, 33, 229 (1976).

6. K. H. Yoon, S. C. Lee, and O. O. Park, Polym. J., 26, 816 (1994).

7. M. Guo and H. G. Zachmann, Polymer, 34, 2503 (1993).

8. I. Ando and G. A. Webb, "Theory of NMR Parameters," Academic Press, New York, N.Y., 1983.

9. A. E. Tonelli and F. C. Schilling, Acc. Chem. Res., 14, 233 (1981).

10. T. Komoto et al., to be published.

11. H. G. Zachmann, D. Wiswe, R. Gehrke, and C. Riekel, Makromol. Chem. Suppl., 12, 175 (1985).

12. T. Sorita, T. Yamanobe, T. Komoto, I. Ando, H. Sato, K. Deguchi, and M. Imanari, Makromol. Chem., Rapid Commun., 5, 657 (1984).

13. I. Ando, T. Yamanobe, T. Sorita, T. Komoto, H. Sato, K. Deguchi, and M. Imanari, Macromolecules, 17, 1995 (1984).

14. T. Yamanobe, T. Sorita, T. Komoto, I. Ando, and H. Sato, J. Mol. Struct., 131, 267 (1985).

15. D. L. VanderHart, J. Magn. Reson., 44, 117 (1981). 